\title{
Quality-Oriented Facade Systems: Technical Data Referred to Maintenance Phase and Residual Value
}

\author{
Rachele Perego \\ School of Architectural Engineering, Politecnico di Milano, Milan 20133, Italy
}

\begin{abstract}
Nowadays, clients require solutions that are efficient during the service life as a whole. In this view, maintenance has functional value and greatly contributes to increasing quality in environments. With reference to maintenance phase and residual value of building products upon dismantling, the present manuscript investigates a set of technological packages of quality and technical value referred to two alternative facade systems available for recovery actions on Italian council buildings- the ETICS (external thermal insulation composite system) and the external cladding system — complying with one thermal-insulation strategy, but different in terms of service life duration. Preservation of technical value and value increases are strictly related to maintenance quality and efficiency. Volume of investments in maintenance is not the only parameter to be considered to assess facade-system-management quality. Time-accurate economic assessment requires the entity to be related to periodical checks on performance quality in the building system and in its parts. Effective technical data to draw up consistent estimates and appraisals are rarely available in literature. With all that stated above, the present manuscript aims at introducing technical data related to drawing up reliable cost estimates and effective appraisals in terms of economics, quality and environmental sustainability. Conclusions will be drawn from the end of the manuscript.
\end{abstract}

Key words: Facade system, service life, maintenance services, residual value, future cost estimate, quality, sustainability.

\section{Introduction}

An analysis applied to upgrading action(s) on an existing building, considering the whole service life of reasonable energy upgrading solution(s), does not only require initial investments to be taken into account $[1,2]$. Technical elements, as well as management measures to reduce energy and environmental consumption [3], could finally be regarded as being able to add value to the reference existing building. Nobody is so naive to think that energy quality is the only thing that counts in a building, and even items related to ordinary maintenance and recovery works are often connected in some way to energy consumption [4]. All energy-saving initiatives tend to shift resources and investments from energy to maintenance. Together

Corresponding author: Rachele Perego, Ph.D., research fields: applied technical architecture, environmental analyses and evaluations, and properties of materials for cultural heritage.E-mail: rachele.perego@polimit.it. with a convincing monetary convenience in funding [5], utility of a reference upgrading solution in terms of quality, sustainability and energy saving should be appraised considering the service life of an entity as a whole $[6,7]$. With reference to the existing building's energy recovery, vertical enclosures represent an important area for system upgrading [8]. Facade systems are complex products. Functions accomplished within facade systems can be maintained during the whole service life, regulated or, if any, measured, only through scheduled maintenance activities. In this view, effective technical data to draw up consistent estimates and appraisals are rarely available in literature. The paper aims at introducing to the reader the effective technical data to draw up reliable future cost estimates and quality-oriented appraisals of a set of technological packages referred to two alternative facade systems-the ETICS (external thermal insulation composite system) $[9,10]$ and the external cladding system [11]—widely used to 
recover outside walls of council buildings in Italy. Detailed technological information concerning analysed solutions is reported in Table 1.

With reference to initial investments, maintenance phase and residual value upon dismantling, the paper will show that economic and environmental indicators for the seventh requirement of building products [12] may be contrasting.

\section{Basic Assumptions: Reasons behind Decision}

In the analysed facade systems (Table 1), the author has kept some layers of system stratigraphy fixed. Only the insulating product has been changed. This assumption has been done by assessing compatibility of coatings and insulating materials in available solutions in advance. Coatings that do not change in the present analysis are widely used for recovery actions in Italian council buildings. In practical terms, the same coating has been kept so that attention can be focused on insulating product's environmental sustainability. In the case of external cladding system, the coating of fibre-reinforced concrete [13] and the anchoring system to posts have been kept fixed, which are often used to recover council building since they are a good compromise between monetary aspects and quantity optimisation without disregarding quality.

In the ETICS, plaster coating is fixed with a siloxane finishing that is compatible both with glass wool and mineral wool and with SEP (sintered expanded

Table 1 Alternative technological solutions.

\begin{tabular}{|c|c|c|}
\hline \multirow{3}{*}{$\begin{array}{l}\text { External thermal } \\
\text { insulation } \\
\text { composite system }\end{array}$} & $\begin{array}{l}\text { Technological } \\
\text { Solution No. } 1\end{array}$ & $\begin{array}{l}\text { (1.1) Siloxane coating (finishing coat) }(\mathrm{F}) \text {. } \\
\text { (1.2) Base coat (together with reinforcement) }(\mathrm{F}) . \\
\text { (1.3) SEP in panels, size: } 100 \times 60 \mathrm{~cm} \text {, thickness: } 8 \mathrm{~cm} \text {. } \\
\text { (1.4) Adhesive bed with additional mechanical fixing — anchoring (F). }\end{array}$ \\
\hline & $\begin{array}{l}\text { Technological } \\
\text { Solution No. } 2\end{array}$ & $\begin{array}{l}\text { (2.1) Siloxane coating (finishing coat) }(\mathrm{F}) \text {. } \\
\text { (2.2) Base coat (together with reinforcement) }(\mathrm{F}) \text {. } \\
\text { (2.3) Rock wool in panels, size: } 100 \times 60 \mathrm{~cm} \text {, thickness: } 8 \mathrm{~cm} \text {. } \\
\text { (2.4) Adhesive bed with additional mechanical fixing - anchoring (F). }\end{array}$ \\
\hline & $\begin{array}{l}\text { Technological } \\
\text { Solution No. } 3\end{array}$ & $\begin{array}{l}\text { (3.1) Siloxane coating (finishing coat) }(\mathrm{F}) \text {. } \\
\text { (3.2) Base coat (together with reinforcement) }(\mathrm{F}) \text {. } \\
\text { (3.3) Glass wool in panels, size: } 100 \times 60 \mathrm{~cm} \text {, thickness: } 7 \mathrm{~cm} \text {. } \\
\text { (3.4) Adhesive bed with additional mechanical fixing - anchoring (F). }\end{array}$ \\
\hline \multirow{2}{*}{$\begin{array}{l}\text { External cladding } \\
\text { system }\end{array}$} & $\begin{array}{l}\text { Technological } \\
\text { Solution No. } 4\end{array}$ & $\begin{array}{l}\text { (4.1) Fibre-reinforced concrete panels, thickness: } 0.8 \mathrm{~cm}(\mathrm{~F}) \text {. } \\
\text { (4.2) Anchoring system (F): (4.2.1) extruded profile in aluminium alloy EN } 6060 \mathrm{~T} 5(\mathrm{~F}) ; \\
\text { (4.2.2) hooks type to support and retain tiles, in stainless steel EN } 14310 \text { (AISI 301) (F); } \\
\text { (4.2.3) springs type to fix hooks on profile, in stainless steel EN } 14310 \text { (AISI 301) (F); (4.2.4) } \\
\text { standard supporting stirrups type, from extruded profile in aluminium alloy EN } 6060 \text { (F); } \\
\text { (4.2.5) nuts and bolts to fix profile on the stirrups, in stainless steel class A2 (F); (4.2.6) } \\
\text { anchors to fix the stirrups on the wall, mechanical or epoxy-resin based, as needed (F); (4.2.7) } \\
\text { neoprene gasket strips, to gauge the tiles, the hooks and the aluminium profile, in different } \\
\text { thickness as needed (F); and (4.2.8) springs in tempered stainless steel, to support the } \\
\text { insulating panels and press them on the wall surface (F). } \\
\text { (4.3) Rock wool in panels, size: } 100 \times 60 \mathrm{~cm} \text {, thickness: } 8 \mathrm{~cm} \text {. } \\
\text { (4.4) Adhesive bed with additional mechanical fixing-anchoring (F). }\end{array}$ \\
\hline & $\begin{array}{l}\text { Technological } \\
\text { Solution No. } 5\end{array}$ & $\begin{array}{l}\text { (5.1) Fibre-reinforced concrete panels; thickness: } 0.8 \mathrm{~cm}(\mathrm{~F}) \text {. } \\
\text { (5.2) Anchoring system (F): (5.2.1) extruded profile in aluminium alloy EN } 6060 \mathrm{~T} 5 \text { (F); } \\
\text { (5.2.2) hooks type to support and retain tiles, in stainless steel EN } 14310 \text { (AISI 301) (F); } \\
\text { (5.2.3) springs type to fix hooks on profile, in stainless steel EN } 14310 \text { (AISI 301) (F); (5.2.4) } \\
\text { standard supporting stirrups type, from extruded profile in aluminium alloy EN } 6060(\mathrm{~F}) ; \\
\text { (5.2.5) nuts and bolts to fix profile on the stirrups, in stainless steel class A2 (F); (5.2.6) } \\
\text { anchors to fix the stirrups on the wall, mechanical or epoxy-resin based, as needed (F); (5.2.7) } \\
\text { neoprene gasket strips, to gauge the tiles, the hooks and the aluminium profile, in different } \\
\text { thickness as needed (F); and (5.2.8) springs in tempered stainless steel, to support the } \\
\text { insulating panels and press them on the wall surface (F). } \\
\text { (5.3) Glass wool in panels; size: } 100 \times 60 \mathrm{~cm} \text {, thickness: } 7 \mathrm{~cm} \text {. } \\
\text { (5.4) Adhesive bed with additional mechanical fixing-anchoring (F). }\end{array}$ \\
\hline
\end{tabular}

(F) means layer of system stratigraphy kept fixed. 
polystyrene). The choice was based on a few reasons. Siloxane plaster coating is of course more expensive compared with organic and silicate coatings which, unlike siloxane coating, are not compatible with the three insulating products used.

Siloxane coatings are also more resistant against UV (ultraviolet) rays compared with an inorganic finishing. Swelling is also lower compared with an organic finishing. As a consequence, as water simply runs off the facade (e.g., rain), dirt accumulating on walls is taken to ground.

In addition, for guaranteeing homogeneity in terms of facade system mapping, insulating product size has been kept constant $(100 \times 60 \mathrm{~cm})$, since it can be effectively used for all the three types of insulating products, for both the external cladding system and the external thermal insulation composite system (a priori fixed dimensional configuration).

\section{Maintenance Phase}

Reliability of a building can be increased by drawing up detailed schedules including inspections, repairs or replacements $[14,15]$. Such schedules involve expenses that are often relevant - the aim is specifying an ideal inspection and repair strategy to minimise costs for building operation. Implementation of a true maintenance policy in existing buildings provides for timely identification of possible anomalies [16], which can be categorised according to the measures to be taken.

Obtained results can help to: (1) update knowledge in terms of current building status and development of degradation; (2) support decisions on preservation, repair, upgrading or functional limitations; (3) in case of conformity, define additional inspections, if required, and schedule them.

\subsection{Towards the Use of a Probability-Based,} Statistical Methodology for Quality Appraisal and Cost Estimate

The problem of optimising occurrence frequencies without a probability-based approach has only found empirical solutions up to now [17]. Such solutions are based on the assumption that assigning frequencies at close ranges provide unreliable information, since no remarkable degradation can develop over a very short period of time [18]. Assigning spars frequencies can make possible repairs impossible, since developed degradation can be excessive. With all that stated above, inspection and maintenance programmes should be drawn up so that their costs can be compensated by useful information and balanced by residual building value. After defining service life, regulated or, if any, measured-ETICS: 30 years [9]; external cladding system: 45 years-frequency of inspection, cleaning and replacement for the two facade systems are included in Tables 2-4. With reference to the non-part research carried out by the author, the schedule has been drawn up by assigning a statistical distribution to each range of terms.

Then, referring to codes of operating instructions of the two facade systems, a maintenance programme of upgrading works (source of inspiration/starting point for subsequent data elaboration [14]) has been set up. A reference monetary value has been estimated for each item in the schedule.

Then, maintenance costs, rebuilding costs, if any, and end-of-life, i.e., dismantling costs actualised to present value, can be calculated based on a maintenance schedule supposed to be drawn up by a provider for a specific block of flats.

With all that stated above, analyses have been carried out on data configurations of all front views for the case study (Fig. 1).

For Fig. 2, we should note that cost actualisation considers building time as time " 0 ". The two compared systems have different service life durations. Cash flows can be grouped by categories of interest. Each of them may qualify as profits $(+)$ or liabilities (-) for the building. Therefore, "-" indicates that maintenance costs, rebuilding costs and end-of-life costs are losses/liabilities in cash flow for works. 
Table 2 External-thermal-insulation-composite-system operating instruction. Inventory of service visits complete with individual instructions and inventory of maintenance services complete with individual instructions.

\begin{tabular}{|c|c|c|c|c|c|c|}
\hline ID & $\begin{array}{l}\text { Inspection type } \\
\text { (ISP) or action } \\
\text { type }(\mathrm{PZ}-\mathrm{RP})^{\mathrm{a}}\end{array}$ & Operating instruction & Operator & Additional notes & $\begin{array}{l}\text { Consequences } \\
\text { for failure to } \\
\text { provide } \\
\text { maintenance } \\
\text { service } \\
\end{array}$ & Frequency \\
\hline ISP1 & $\begin{array}{l}\text { Surface } \\
\text { inspection }\end{array}$ & $\begin{array}{l}\text { Please inspect from at least } \\
\text { a 3-meter standpoint under } \\
\text { normal daylight. Do not } \\
\text { inspect under direct sunlight }\end{array}$ & $\begin{array}{l}\text { Tenant. } \\
\text { Qualified } \\
\text { personnel }\end{array}$ & See specific case & $\begin{array}{l}\text { Deteriorated } \\
\text { aesthetics. } \\
\text { Altered } \\
\text { performance }\end{array}$ & $\begin{array}{l}\text { Cannot be } \\
\text { defined } \\
\text { apriori. } \\
\text { Cannot be } \\
\text { generalised } \\
\end{array}$ \\
\hline ISP2 & $\begin{array}{l}\text { Run a plaster } \\
\text { test to verify } \\
\text { state of } \\
\text { preservation }\end{array}$ & $\begin{array}{l}\text { Sight and/or } \\
\text { instrument-aided analysis }\end{array}$ & $\begin{array}{l}\text { Qualified } \\
\text { personnel } \\
\text { only }\end{array}$ & See specific case & $\begin{array}{l}\text { Altered } \\
\text { performance. } \\
\text { Malfunctioning }\end{array}$ & $\begin{array}{l}\text { Cannot be } \\
\text { defined } \\
\text { apriori. } \\
\text { Cannot be } \\
\text { generalised }\end{array}$ \\
\hline ISP3 & $\begin{array}{l}\text { Assessment of } \\
\text { any damage } \\
\text { caused by } \\
\text { predictable } \\
\text { increased stress }\end{array}$ & $\begin{array}{l}\text { Sight and/or } \\
\text { instrument-aided analysis }\end{array}$ & $\begin{array}{l}\text { Qualified } \\
\text { personnel } \\
\text { only }\end{array}$ & $\begin{array}{l}\text { Check for cracks or moulds on } \\
\text { weather-exposed walls. Check } \\
\text { finished surface especially where } \\
\text { most susceptible to vandalism. } \\
\text { Check for water infiltration near } \\
\text { rain gutters, downspouts or ground } \\
\text { connection. Check for any break-up, } \\
\text { detachment or fissuring near } \\
\text { thermal bridges and joints }\end{array}$ & $\begin{array}{l}\text { Deteriorated } \\
\text { aesthetics. } \\
\text { Altered } \\
\text { performance. } \\
\text { Malfunctioning }\end{array}$ & $\begin{array}{l}\text { Cannot be } \\
\text { defined } \\
\text { apriori. } \\
\text { Cannot be } \\
\text { generalised }\end{array}$ \\
\hline ISP4 & $\begin{array}{l}\text { Assessment of } \\
\text { surface damage } \\
\text { due to } \\
\text { unpredictable } \\
\text { events } \\
\end{array}$ & $\begin{array}{l}\text { Sight and/or } \\
\text { instrument-aided analysis }\end{array}$ & $\begin{array}{l}\text { Qualified } \\
\text { personnel } \\
\text { only }\end{array}$ & See specific case & $\begin{array}{l}\text { See specific } \\
\text { case }\end{array}$ & $\begin{array}{l}\text { Cannot be } \\
\text { defined } \\
\text { apriori. } \\
\text { Cannot be } \\
\text { generalised } \\
\end{array}$ \\
\hline ISP5 & $\begin{array}{l}\text { Control of any } \\
\text { inducted } \\
\text { structural } \\
\text { damage }\end{array}$ & $\begin{array}{l}\text { Sight and/or } \\
\text { instrument-aided analysis }\end{array}$ & $\begin{array}{l}\text { Qualified } \\
\text { personnel } \\
\text { only }\end{array}$ & See specific case & $\begin{array}{l}\text { See specific } \\
\text { case }\end{array}$ & $\begin{array}{l}\text { Cannot be } \\
\text { defined } \\
\text { apriori. } \\
\text { Cannot be } \\
\text { generalised } \\
\end{array}$ \\
\hline ISP6 & $\begin{array}{l}\text { Check for any } \\
\text { execution and/or } \\
\text { maintenance } \\
\text { flaws }\end{array}$ & $\begin{array}{l}\text { Sample sight and/or } \\
\text { instrument-aided analysis. } \\
\text { See specific case }\end{array}$ & $\begin{array}{l}\text { Qualified } \\
\text { personnel } \\
\text { only }\end{array}$ & $\begin{array}{l}\text { Inspection to be carried out during } \\
\text { dismantling of large portions of the } \\
\text { façade system or during first full } \\
\text { maintenance service. See specific } \\
\text { case }\end{array}$ & - & $\begin{array}{l}30 \text { years } \\
\text { (measured } \\
\text { service life) }\end{array}$ \\
\hline PZ1 & $\begin{array}{l}\text { Extraordinary } \\
\text { cleaning }\end{array}$ & $\begin{array}{l}\text { Wash finished surface with } \\
\text { water }\end{array}$ & $\begin{array}{l}\text { Qualified } \\
\text { personnel } \\
\text { only }\end{array}$ & $\begin{array}{l}\text { Before washing, remove any stains, } \\
\text { including any hard scale build-up, } \\
\text { and graffiti by brushing off any } \\
\text { grease build-up either chemically or } \\
\text { mechanically }\end{array}$ & $\begin{array}{l}\text { Surface } \\
\text { deterioration. } \\
\text { Deteriorated } \\
\text { aesthetics }\end{array}$ & 15 years \\
\hline RP1 & $\begin{array}{l}\text { Restore surface } \\
\text { where necessary. } \\
\text { Replace elements } \\
\text { which are most } \\
\text { susceptible to } \\
\text { wear, breakage } \\
\text { or decay. } \\
\end{array}$ & $\begin{array}{l}\text { Restore locally by covering } \\
\text { with ready-to-use } \\
\text { cement-free base coat. No } \\
\text { reinforcement or finishing } \\
\text { coat necessary. }\end{array}$ & $\begin{array}{l}\text { Qualified } \\
\text { personnel } \\
\text { only }\end{array}$ & Restoring causing uneven surface. & $\begin{array}{l}\text { Surface } \\
\text { deterioration. } \\
\text { Deteriorated } \\
\text { aesthetics }\end{array}$ & $\begin{array}{l}\text { Cannot be } \\
\text { defined } \\
\text { apriori. } \\
\text { Cannot be } \\
\text { generalised }\end{array}$ \\
\hline RP2 & $\begin{array}{l}\text { Reapply surface } \\
\text { finishing coat }\end{array}$ & $\begin{array}{l}\text { Clean and prepare support } \\
\text { by using hardeners, primers, } \\
\text { or by applying a base coat } \\
\text { always bearing in mind the } \\
\text { chemical composition of the } \\
\text { paint to be used. } \\
\text { Apply two or more coats of } \\
\text { paint }\end{array}$ & $\begin{array}{l}\text { Qualified } \\
\text { personnel } \\
\text { only }\end{array}$ & $\begin{array}{l}\text { Use same type of pre-existing paint } \\
\text { or analogous product in accordance } \\
\text { with manufacturer's instructions }\end{array}$ & $\begin{array}{l}\text { Surface } \\
\text { deterioration. } \\
\text { Deteriorated } \\
\text { aesthetics }\end{array}$ & $\begin{array}{l}\text { Cannot be } \\
\text { defined } \\
\text { apriori. } \\
\text { Cannot be } \\
\text { generalised }\end{array}$ \\
\hline
\end{tabular}


(continued Table 2)

\begin{tabular}{|l|l|l|l|l|l|l|}
\hline ID & $\begin{array}{l}\text { Inspection type } \\
\text { (ISP) or action } \\
\text { type (PZ-RP) }\end{array}$ & Operating instruction & Operator & Additional notes & $\begin{array}{l}\text { Consequences } \\
\text { for failure to } \\
\text { provide } \\
\text { maintenance } \\
\text { service }\end{array}$ & Frequency \\
\hline RP3 & Remove & Dismantle the facade system & $\begin{array}{l}\text { Qualified } \\
\text { personnel } \\
\text { only }\end{array}$ & - & - & 30 years \\
\hline
\end{tabular}

aThe column titled "Inspection type (ISP) or action type (PZ-RP)" has been elaborated starting from Ref. [14].

Table 3 External-cladding-system operating instruction. Inventory of service visits complete with individual instructions.

\begin{tabular}{|c|c|c|c|c|c|}
\hline ID & $\begin{array}{l}\text { Inspection type } \\
\text { (ISP) or action } \\
\text { type }(\mathrm{PZ}-\mathrm{RP})^{\mathrm{a}}\end{array}$ & $\begin{array}{l}\text { Operating instruction } \\
\text { operator }\end{array}$ & Additional notes & $\begin{array}{l}\text { Consequences for } \\
\text { failure to provide } \\
\text { maintenance } \\
\text { service }\end{array}$ & Frequency \\
\hline ISP7 & Surface inspection & $\begin{array}{l}\text { Sight inspection to be carried out by } \\
\text { tenant. Please inspect from at least a } \\
\text { 3-meter standpoint under normal } \\
\text { daylight. Do not inspect under direct } \\
\text { sunlight }\end{array}$ & See specific case & $\begin{array}{l}\text { Deteriorated } \\
\text { aesthetics. } \\
\text { Altered } \\
\text { performance }\end{array}$ & $\begin{array}{l}\text { Cannot be } \\
\text { generalised }\end{array}$ \\
\hline ISP8 & $\begin{array}{l}\text { Inspection of } \\
\text { open-jointed wall } \\
\text { cladding system }\end{array}$ & $\begin{array}{l}\text { Sample sight and/or instrument-aided } \\
\text { analysis to be carried out in the most } \\
\text { critically exposed areas by qualified } \\
\text { personnel only. See specific case }\end{array}$ & $\begin{array}{l}\text { Please ensure that all joints } \\
\text { located in critical spots are } \\
\text { firmly secured to allow runoff } \\
\text { water to flow away from both } \\
\text { external and internal wall } \\
\text { sidings, thus avoiding any } \\
\text { contact with the wall } \\
\text { substructure }\end{array}$ & $\begin{array}{l}\text { Altered } \\
\text { performance. } \\
\text { Malfunctioning }\end{array}$ & $\begin{array}{l}\text { Cannot be } \\
\text { generalised }\end{array}$ \\
\hline ISP9 & $\begin{array}{l}\text { Assessment of } \\
\text { expansion anchors }\end{array}$ & $\begin{array}{l}\text { Sample sight and/or instrument-aided } \\
\text { analysis to be carried out by qualified } \\
\text { personnel only. See specific case }\end{array}$ & See specific case & See specific case & $\begin{array}{l}\text { Cannot be } \\
\text { generalised }\end{array}$ \\
\hline ISP10 & $\begin{array}{l}\text { Assessment of any } \\
\text { wall cladding } \\
\text { damage caused by } \\
\text { predictable } \\
\text { increased stres } \\
\end{array}$ & $\begin{array}{l}\text { Sample sight and/or instrument-aided } \\
\text { analysis to be carried out by qualified } \\
\text { personnel only. See specific case }\end{array}$ & See specific case & See specific case & $\begin{array}{l}\text { Cannot be } \\
\text { generalised }\end{array}$ \\
\hline ISP11 & $\begin{array}{l}\text { Assessment of } \\
\text { surface damage } \\
\text { due to } \\
\text { unpredictable } \\
\text { events }\end{array}$ & $\begin{array}{l}\text { Sample sight and/or instrument-aided } \\
\text { analysis to be carried out by qualified } \\
\text { personnel only. See specific case }\end{array}$ & $\begin{array}{l}\text { Assessment to be carried out } \\
\text { after the occurrence of an } \\
\text { unforeseeable incident. See } \\
\text { specific case }\end{array}$ & $\begin{array}{l}\text { Altered } \\
\text { performance }\end{array}$ & $\begin{array}{l}\text { Cannot be } \\
\text { generalised }\end{array}$ \\
\hline ISP12 & $\begin{array}{l}\text { Check for any } \\
\text { execution and/or } \\
\text { maintenance flaws }\end{array}$ & $\begin{array}{l}\text { Sample sight and/or instrument-aided } \\
\text { analysis to be carried out by qualified } \\
\text { personnel only. See specific case }\end{array}$ & $\begin{array}{l}\text { Inspection to be carried out } \\
\text { during dismantling of large } \\
\text { portions of the facade or during } \\
\text { first full maintenance service. } \\
\text { See specific case }\end{array}$ & - & 45 years \\
\hline
\end{tabular}

${ }^{a}$ The column titled "Inspection type (ISP) or action type (PZ-RP)" has been elaborated starting from Ref. [14].

Table 4 External-cladding-system operating instruction. Inventory of maintenance services complete with individual instructions.

\begin{tabular}{|l|l|l|l|l|l|}
\hline ID & $\begin{array}{l}\text { Inspection type (ISP) } \\
\text { or action type } \\
(\text { PZ-RP) }\end{array}$ & $\begin{array}{l}\text { Operating instruction and } \\
\text { operator }\end{array}$ & Additional notes & $\begin{array}{l}\text { Consequences for } \\
\text { failure to provide } \\
\text { maintenance service }\end{array}$ & Frequency \\
\hline PZ2 & $\begin{array}{l}\text { Routine cleaning. } \\
\begin{array}{l}\text { Wall exteriors must } \\
\text { be hydroblasted } \\
\text { whenever dust } \\
\text { build-up is detected }\end{array}\end{array}$ & $\begin{array}{l}\text { Wash using a fan-shaped } \\
\text { nozzled hydro jet blaster (max. } \\
\text { pressure: 80 bar; minimum } \\
\text { distance from wall cladding: 25 } \\
\mathrm{cm})\end{array}$ & $\begin{array}{l}\text { Before proceeding with the } \\
\text { clean-up, always run a test on a } \\
\text { non-visible side of the cladding } \\
\text { panel. Never clean the wall facing in } \\
\text { broad daylight. Wall systems and } \\
\text { wall cladding panels should not be } \\
\text { dry-cleaned using chemical solvents }\end{array}$ & $\begin{array}{l}\text { Surface deterioration. } \\
\text { Deteriorated } \\
\text { aesthetics }\end{array}$ & $10-15$ years \\
\hline
\end{tabular}


(continued Table 4)

\begin{tabular}{|c|c|c|c|c|c|}
\hline ID & $\begin{array}{l}\text { Inspection type (ISP) } \\
\text { or action type } \\
(\mathrm{PZ}-\mathrm{RP})^{\mathrm{a}}\end{array}$ & $\begin{array}{l}\text { Operating instruction and } \\
\text { operator }\end{array}$ & Additional notes & $\begin{array}{l}\text { Consequences for } \\
\text { failure to provide } \\
\text { maintenance service }\end{array}$ & Frequency \\
\hline PZ3 & $\begin{array}{l}\text { Removal of surface } \\
\text { debris. } \\
\text { Wet dust clean-up } \\
\text { and removal }\end{array}$ & $\begin{array}{l}\text { Wash abundantly with water using } \\
\text { a sponge or a soft brush }\end{array}$ & $\begin{array}{l}\text { Wet dust can stain the wall } \\
\text { cladding surface }\end{array}$ & $\begin{array}{l}\text { Surface deterioration. } \\
\text { Deteriorated } \\
\text { aesthetics }\end{array}$ & $\begin{array}{l}\text { Cannot be } \\
\text { generalised }\end{array}$ \\
\hline PZ4 & $\begin{array}{l}\text { Removal of surface } \\
\text { debris. } \\
\text { Dry dust clean-up } \\
\text { and removal }\end{array}$ & $\begin{array}{l}\text { Vacuum clean the area. } \\
\text { Alternatively, use a clean dry } \\
\text { cloth or brush }\end{array}$ & - & $\begin{array}{l}\text { Surface deterioration. } \\
\text { Deteriorated } \\
\text { aesthetics }\end{array}$ & $\begin{array}{l}\text { Cannot be } \\
\text { generalised }\end{array}$ \\
\hline PZ5 & $\begin{array}{l}\text { Removal of surface } \\
\text { debris. } \\
\text { Clean-up and } \\
\text { removal of non-hard } \\
\text { water stains }\end{array}$ & $\begin{array}{l}\text { Jet blast with cold water (max. } \\
\text { pressure: } 80 \text { bar; minimum distance } \\
\text { from wall cladding: } 25 \mathrm{~cm} \text { ). Use a } \\
\text { fan-shaped nozzled jet sprayer }\end{array}$ & $\begin{array}{l}\text { Before proceeding, run a test on the } \\
\text { back side of the cladding panel. Do } \\
\text { not use detergents to clean the } \\
\text { panes. Do not wash those cladding } \\
\text { panels exposed to the heat of the } \\
\text { sunshine with alkaline or acid } \\
\text { products since these can leave stains }\end{array}$ & $\begin{array}{l}\text { Surface deterioration. } \\
\text { Deteriorated } \\
\text { aesthetics }\end{array}$ & $\begin{array}{l}\text { Cannot be } \\
\text { generalised }\end{array}$ \\
\hline PZ6 & $\begin{array}{l}\text { Removal of surface } \\
\text { debris. } \\
\text { Clean-up and } \\
\text { removal of } \\
\text { hard-water stains }\end{array}$ & $\begin{array}{l}\text { (1) Spray coat in acetic acid } \\
\text { solution }(10 \%) ;(2) \text { allow the } \\
\text { solution to rest for a few minutes. } \\
\text { Do not let it dry out; (3) rinse the } \\
\text { cladding in water (see PZ4); (4) } \\
\text { repeat stage (1) through (3) for } \\
\text { more stubborn stains }\end{array}$ & $\begin{array}{l}\text { Do not use detergents to clean the } \\
\text { panes. Do not wash those cladding } \\
\text { panels exposed to the heat of the } \\
\text { sunshine with alkaline or acid } \\
\text { products since these can leave } \\
\text { stains }\end{array}$ & $\begin{array}{l}\text { Surface deterioration. } \\
\text { Deteriorated } \\
\text { aesthetics }\end{array}$ & $\begin{array}{l}\text { Cannot be } \\
\text { generalised }\end{array}$ \\
\hline PZ7 & $\begin{array}{l}\text { Removal of surface } \\
\text { debris. } \\
\text { Clean-up and } \\
\text { removal of concrete } \\
\text { acid stains }\end{array}$ & $\begin{array}{l}\text { (1) Remove concrete stains } \\
\text { immediately by applying the } \\
\text { acetic acid solution }(10 \%) ;(2) \text { let } \\
\text { it stand for } 5 \text { to } 20 \text { minutes. Do } \\
\text { not let it dry out; }(3) \text { rinse in clean } \\
\text { cold water using a high-pressure } \\
\text { water jet }(40 \div 80 \text { bar) }\end{array}$ & $\begin{array}{l}\text { Before proceeding with the } \\
\text { clean-up, always run a test on a } \\
\text { non-visible side of the cladding } \\
\text { panel }\end{array}$ & $\begin{array}{l}\text { Surface deterioration. } \\
\text { Deteriorated } \\
\text { aesthetics }\end{array}$ & $\begin{array}{l}\text { Cannot be } \\
\text { generalised }\end{array}$ \\
\hline PZ8 & $\begin{array}{l}\text { Removal of surface } \\
\text { debris. } \\
\text { Clean-up and } \\
\text { removal of graffiti }\end{array}$ & $\begin{array}{l}\text { The best option is to replace the } \\
\text { stained cladding panels. As a } \\
\text { matter of fact, anti-graffiti } \\
\text { coatings or any other special } \\
\text { treatment can change the overall } \\
\text { look of the cladding panels as the } \\
\text { surface can become shinier }\end{array}$ & $\begin{array}{l}\text { Please ask your wall cladding } \\
\text { supplier for advice before } \\
\text { proceeding with the clean-up. Any } \\
\text { inappropriate treatment will result } \\
\text { in your warranty being voided. } \\
\text { Please ask your anti-graffiti } \\
\text { coating manufacturer to provide } \\
\text { adequate insurance }\end{array}$ & $\begin{array}{l}\text { Surface deterioration. } \\
\text { Deteriorated } \\
\text { aesthetics }\end{array}$ & $\begin{array}{l}\text { Cannot be } \\
\text { generalised }\end{array}$ \\
\hline PZ9 & $\begin{array}{l}\text { Routine cleaning of } \\
\text { joints and seals }\end{array}$ & $\begin{array}{l}\text { Use products which are suitable } \\
\text { for the cladding material }\end{array}$ & 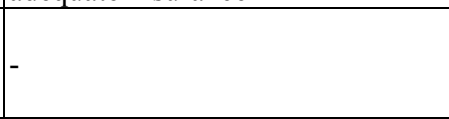 & \begin{tabular}{|l} 
Deteriorated \\
structural joints. \\
Altered performance \\
\end{tabular} & $\begin{array}{l}\text { Cannot be } \\
\text { generalised }\end{array}$ \\
\hline RP4 & $\begin{array}{l}\text { Replacement of } \\
\text { cladding elements, } \\
\text { seals and joints }\end{array}$ & $\begin{array}{l}\text { (1) Dismantle damaged elements; } \\
\text { (2) replace irreparably damaged } \\
\text { elements and either partially or } \\
\text { totally restore joints and seals; (3) } \\
\text { place any missing elements } \\
\text { accordingly }\end{array}$ & See specific case & $\begin{array}{l}\text { Altered performance. } \\
\text { Deteriorated } \\
\text { aesthetics }\end{array}$ & $\begin{array}{l}\text { Cannot be } \\
\text { generalised }\end{array}$ \\
\hline RP5 & $\begin{array}{l}\text { Restore coplanarity } \\
\text { of wall cladding } \\
\text { panels }\end{array}$ & $\begin{array}{l}\text { (1) Disassemble non-coplanar } \\
\text { elements; (2) restore anchoring } \\
\text { system; (3) assemble to the } \\
\text { highest standards of workmanship }\end{array}$ & See specific case & $\begin{array}{l}\text { Altered performance. } \\
\text { Deteriorated } \\
\text { aesthetics }\end{array}$ & $10-15$ years \\
\hline RP6 & \begin{tabular}{|l|} 
Restore existing \\
anchoring structure
\end{tabular} & $\begin{array}{l}\text { Secure bolted joints and restore } \\
\text { existing system }\end{array}$ & See specific case & Altered performance & 45 years \\
\hline $\mathrm{RP7}$ & $\begin{array}{l}\text { Replace existing } \\
\text { anchoring structure }\end{array}$ & Replace damaged anchors & See specific case & Altered performance & 45 years \\
\hline RP8 & Dismantle & $\begin{array}{l}\text { Dismantle structural facade and } \\
\text { cladding }\end{array}$ & - & - & 45 years \\
\hline
\end{tabular}

aThe column titled "Inspection type (ISP) or action type (PZ-RP)" has been elaborated starting from Ref. [14]. 


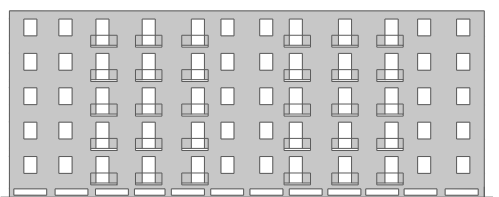

(a)

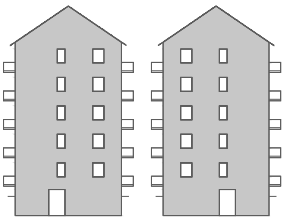

(b)

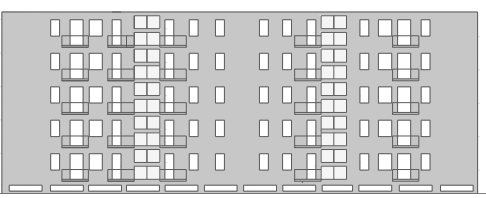

(c)

Fig. 1 The case study. Geometrical configurations: (a) Front View No. 1; (b) Front View No. 2 equals Front View No. 4; (c) Front View No. 3.

Front View No. 1: wall surface: $605.071 \mathrm{~m}^{2}$. ETICS: involved surface: $553.304 \mathrm{~m}^{2}$ of insulating panels, scrap excluded; $719.881 \mathrm{~m}^{2}$ of insulating panels, scrap included. External cladding system: involved surface: $657.709 \mathrm{~m}^{2}$ of reinforced-concrete panels, laid;

Front View No. 2 = Front View No. 4: wall surface: $237.624 \mathrm{~m}^{2}$ (each). ETICS: involved surface: 207.689 (each) $\mathrm{m}^{2}$ of insulating panels, scrap excluded; 224.489 (each) $\mathrm{m}^{2}$ of insulating panels, scrap included. External cladding system: 208.041 (each) $\mathrm{m}^{2} \mathrm{of}$ reinforced-concrete panels, laid;

Front View No. 3: wall surface: $625.629 \mathrm{~m}^{2}$. ETICS: involved surface: $596.703 \mathrm{~m}^{2}$ of insulating panels, scrap excluded; $629.761 \mathrm{~m}^{2}$ of insulating panels, scrap included. External cladding system: $530.297 \mathrm{~m}^{2}$ of reinforced-concrete panels, laid.

\subsection{Environmental Impact}

Consumptions referred to maintenance services have been calculated to appraise environmental impact during the maintenance phase.

Please consider $1 \mathrm{~m}^{2}$ of finished surface to clean and please refer to the following maintenance services:

(1) Extraordinary cleaning of plaster coating fixed with a siloxane finishing. A-priori assignable frequency is equal to 15 years (Monte Carlo Simulation processed by the author (input data: frequency ranges reported in Table 2) $)^{1}$;

(2) Routine cleaning of fibre-reinforced concrete panels, thickness: $0.8 \mathrm{~cm}$, density: $1.9 \mathrm{~g} / \mathrm{cm}^{3}$. A-priori assignable frequency is equal to 13 years (always Monte Carlo Simulation processed by the author (input data: frequency ranges reported in Tables 3 and 4) $)^{2}$.

Since the aforementioned operations have an a-priori assignable frequency, environmental impacts due to water consumptions and diesel consumptions to clean the whole finished surface of the case study have been reasonably appraised. To appraise water consumptions and diesel consumptions, medium

\footnotetext{
${ }^{1}$ Code PZ1, Table 2: involved surface percentage: $100 \%$. Painting is not required after extraordinary cleaning.

${ }^{2}$ Code PZ2, Table 4: routine cleaning (affected surface: $100 \%$ ); RP5, Table 4: restoring co-planarity of wall-cladding panels (assumed affected surface: 5\%). Both activities have been unified so that they can be carried out at the same time, thus, optimising cost for site clearing work needed to carry out both maintenance activities.
}

values between industrial and non-industrial high-pressure water jet machines have been defined ${ }^{3}$. According to the state of the art, if an industrial high-pressure water jet machine is used to clean the finished surface, $15-16 \mathrm{~L} / \mathrm{m}^{2}$ are supplied. If a non-industrial high-pressure water jet machine is used to clean the finished surface, $1.8 \mathrm{~L} / \mathrm{m}^{2}$ to $2.5 \mathrm{~L} / \mathrm{m}^{2}$ are supplied. For the present analysis, the use of a completely autonomous high-pressure water jet machine from an external source of energy has been hypothesized.

Therefore, such machine runs with spark-ignition or diesel engine and, according to machine dimensions, has manual or electrical chokes supplied by petrol or diesel fuel contained in a tank.

It should be noted that a consumption of the diesel equal to $0.3 \mathrm{~L} / \mathrm{m}^{2}$ has been formulated.

It also should be noted that a standard operational team of workers takes, e.g.:

(1) from 2 to $3 \mathrm{~min} / \mathrm{m}^{2}$ to remove easy stains;

(2) from 5 to $7 \mathrm{~min} / \mathrm{m}^{2}, 10 \mathrm{~min} / \mathrm{m}^{2}$ in some cases, to remove stubborn stains.

To remove the latter types of stains, minimum

\footnotetext{
${ }^{3}$ Data about high-pressure water jet machines with different weight usable to clean the finished surface have been collected during the state of the art. It is hereby pointed out that most appropriate machine usable for specific purposes is not definable a priori. The choice of the most appropriate machine depends on the maintenance service of the surface. As a matter of fact, some high-pressure water jet machines have too much elevated power for some maintenance services.
} 
consumption of detergents is also needed.

Detergents consumptions have been omitted because they cannot be generalized and defined apriori because quantity and types of detergents depend on specific stain that occurs on the finished surface.

Results refer to a single maintenance service. Data should be multiplied by the number of times that the maintenance service occurs during the service life of the reference solution as a whole.

Alternative solutions used in the two facade systems, which have been compared by means of two indicators, are well known and widely used to assess global environmental impact of each solution [19].

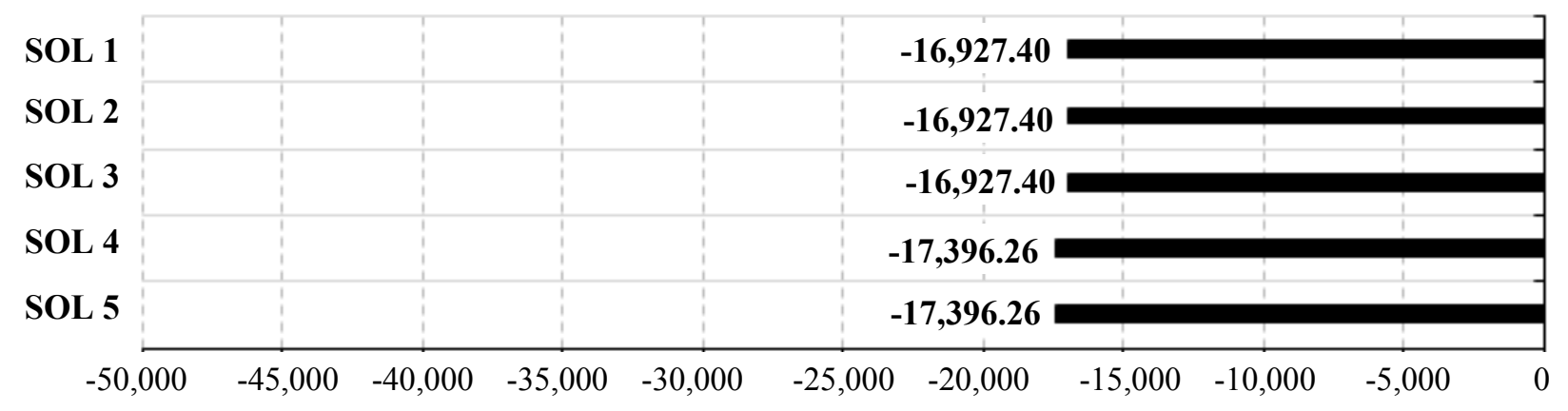

Actualised maintenance costs $(€)$

(a)

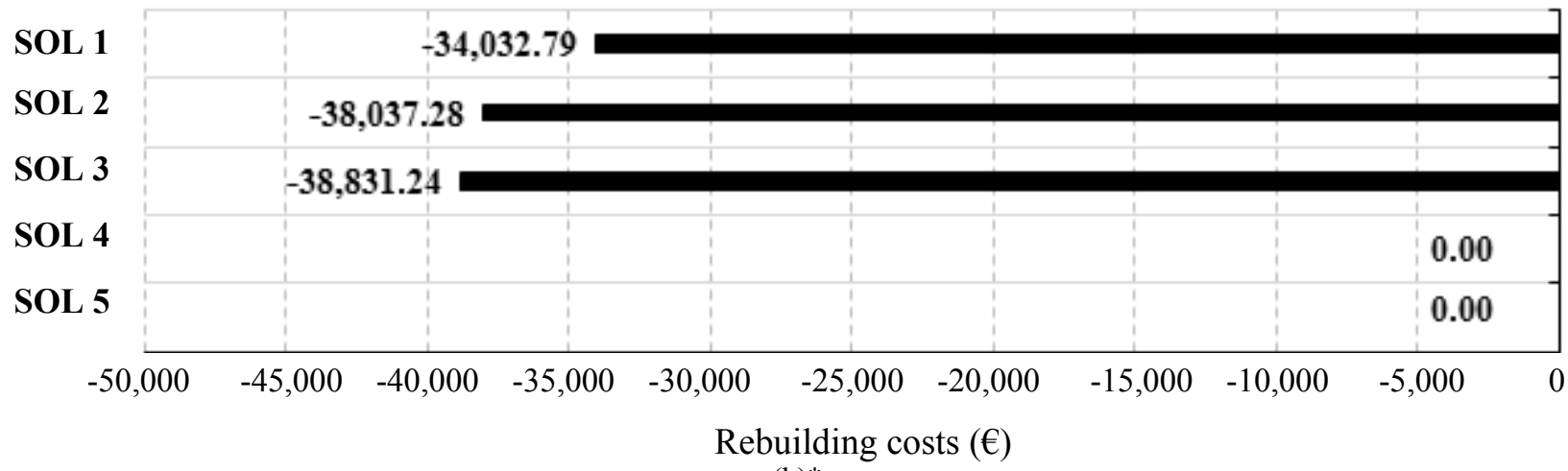

(b)*

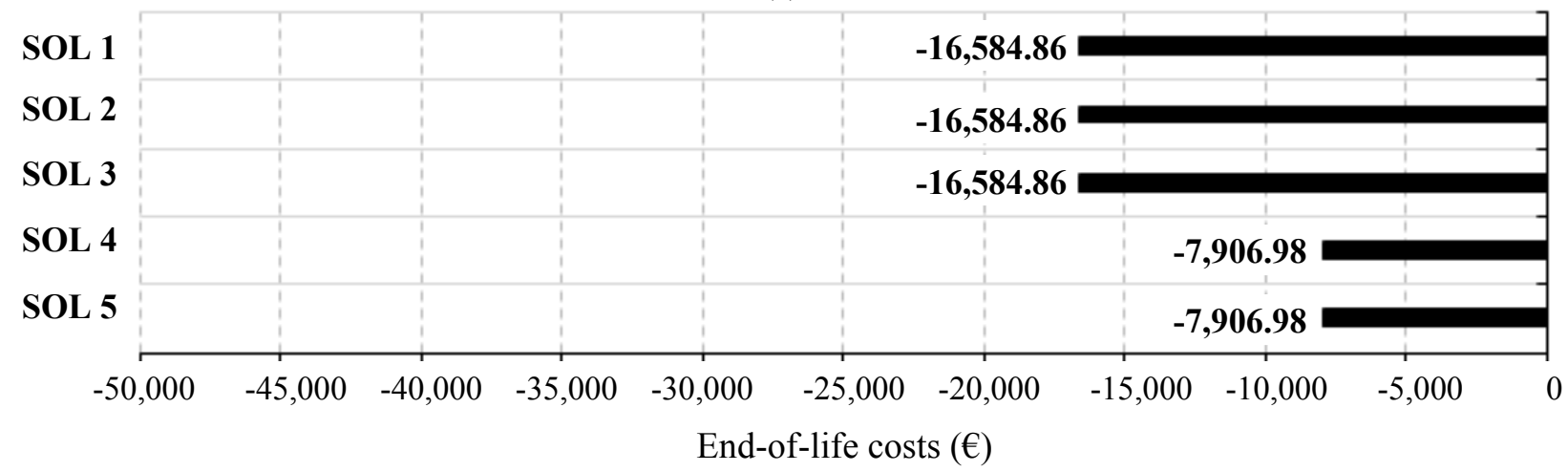

(c)

Fig. 2 Actualised costs (EUR): (a) actualised maintenance costs (EUR); (b) actualised rebuilding costs (EUR); (c) actualised end-of-life (dismantling) costs (EUR).

Reference is made to the case study (Fig. 1). Codes refer to Table 1;

*Dismantling and dismissal of the ETICS is assumed to be after 30 years, with rebuilding at the end of its measured service life. After 45 years, the external cladding system will be dismantled and dismissed, while the new ETICS already rebuilt will require maintenance according to the introduced schedules (Tables 2-4). Therefore, the ETICS will also imply system rebuilding costs. 
The author has tried to cast with a critical eye on the EU objective related to emission into the atmosphere of gases with high global-warming potential and to energy-consumption containments. At this proposal, indicators used are: (1) GWP (global warming potential); (2) EE (embodied energy).

With reference to the analyses carried out, SimaPro (System for Integrated Environmental Assessment of Products) is the assumed databank for collecting data concerning environmental impacts on functional unit.

Obtained results concerning GWP and EE are quantitative (Table 5 and Figs. 3 and 4).

\section{Residual Value upon Dismantling}

Based on researches carried out by the author on the Italian market, solution described in Table 1 can be also compared in terms of residual value.

In terms of residual value, which, of course, can hardly be identified, results are qualitative for both facade systems but show that combinations including insulating materials with lower environmental impact are not those which, in economic terms, have the highest residual value and are the most reusable and recyclable.

Table 5 Compared behaviours of alternative solutions in terms of GWP ( $\mathrm{kg} \mathrm{CO} 2$ equivalent) and of EE (MJ equivalent).

\begin{tabular}{|c|c|c|c|c|c|c|c|c|c|c|}
\hline \multirow{3}{*}{ Description } & \multirow{2}{*}{\multicolumn{2}{|c|}{$\begin{array}{c}\text { Consumption per unit of } \\
\text { measure } \\
\left(\mathrm{L} / \mathrm{m}^{2}\right)\end{array}$}} & \multirow{3}{*}{$\begin{array}{l}\text { Involved } \\
\text { surface } \\
\left(\mathrm{m}^{2}\right)\end{array}$} & \multirow{3}{*}{$\begin{array}{l}\text { Reference } \\
\text { solutions, } \\
\text { Table } 1\end{array}$} & \multirow{2}{*}{\multicolumn{2}{|c|}{ Amount (liter) }} & \multicolumn{4}{|c|}{ Environmental impact on functional unit } \\
\hline & & & & & & & \multicolumn{2}{|c|}{ ( $\mathrm{kg} \mathrm{CO}_{2}$ equivalent) } & \multicolumn{2}{|c|}{ (MJ equivalent) } \\
\hline & Water & Diesel & & & Water & Diesel & Water & Diesel & Water & Diesel \\
\hline \multirow{2}{*}{$\begin{array}{l}\text { Extraordinary } \\
\text { cleaning of plaster } \\
\text { coating fixed with a } \\
\text { siloxane finishing. } \\
\text { Code: PZ1, Table } 2 .\end{array}$} & $\begin{array}{l}\text { Consumptions } \\
15.50 \mathrm{l} / \mathrm{min} \mathbf{~} \\
2.15 \mathrm{l} / \mathrm{min} \mathbf{\Delta}\end{array}$ & \multirow{2}{*}{0.3} & \multirow{2}{*}{$1,675.72$} & \multirow{2}{*}{$\begin{array}{l}1 ; 2 ; 3 \\
\text { ETICS }\end{array}$} & \multirow{2}{*}{$73,941.10$} & \multirow{2}{*}{502.72} & \multirow{2}{*}{0.0000766} & \multirow{2}{*}{0.4277177} & \multirow[b]{2}{*}{0.0006226} & \multirow{2}{*}{52.610119} \\
\hline & $\begin{array}{l}\text { Time } \\
2.50 \mathrm{~min} / \mathrm{m}^{2} \\
7.50 \mathrm{~min} / \mathrm{m}^{2} \boldsymbol{\Delta}\end{array}$ & & & & & & & & & \\
\hline \multirow{2}{*}{$\begin{array}{l}\text { Routine cleaning of } \\
\text { fibre-reinforced } \\
\text { concrete panels, } \\
\text { thickness: } 0.8 \mathrm{~cm} ; \\
\text { density: } 1.9 \mathrm{~g} / \mathrm{cm}^{3} \text {. } \\
\text { Code: PZ2, Table } 4 .\end{array}$} & $\begin{array}{l}\text { Consumptions } \\
15.50 \mathrm{l} / \mathrm{min} \mathbf{} \\
2.15 \mathrm{l} / \mathrm{min} \boldsymbol{\Delta}\end{array}$ & \multirow[b]{2}{*}{0.3} & \multirow{2}{*}{$1,604.09$} & \multirow{2}{*}{$\begin{array}{l}4 ; 5 \\
\text { external } \\
\text { cladding } \\
\text { system }\end{array}$} & \multirow{2}{*}{$70,780.32$} & \multirow{2}{*}{481.23} & \multirow{2}{*}{0.0000766} & \multirow{2}{*}{0.4277177} & \multirow{2}{*}{0.0006226} & \multirow{2}{*}{52.610119} \\
\hline & $\begin{array}{l}\text { Time } \\
2.50 \mathrm{~min} / \mathrm{m}^{2} \\
7.50 \mathrm{~min} / \mathrm{m}^{2} \boldsymbol{\Delta}\end{array}$ & & & & & & & & & \\
\hline
\end{tabular}

Codes refer to Table 1 and data refer to a single maintenance service;

The - square indicates a medium value related to consumptions and times of cleaning introduced in Section 3.2. Data refer to an industrial high-pressure water jet machine. The $\boldsymbol{\Delta}$ triangle indicates a medium value related to consumptions and times of cleaning always introduced in Section 3.2. Data refer to a non-industrial high-pressure water jet machine.

Source: SimaPro-System for Integrated Environmental Assessment of Products.

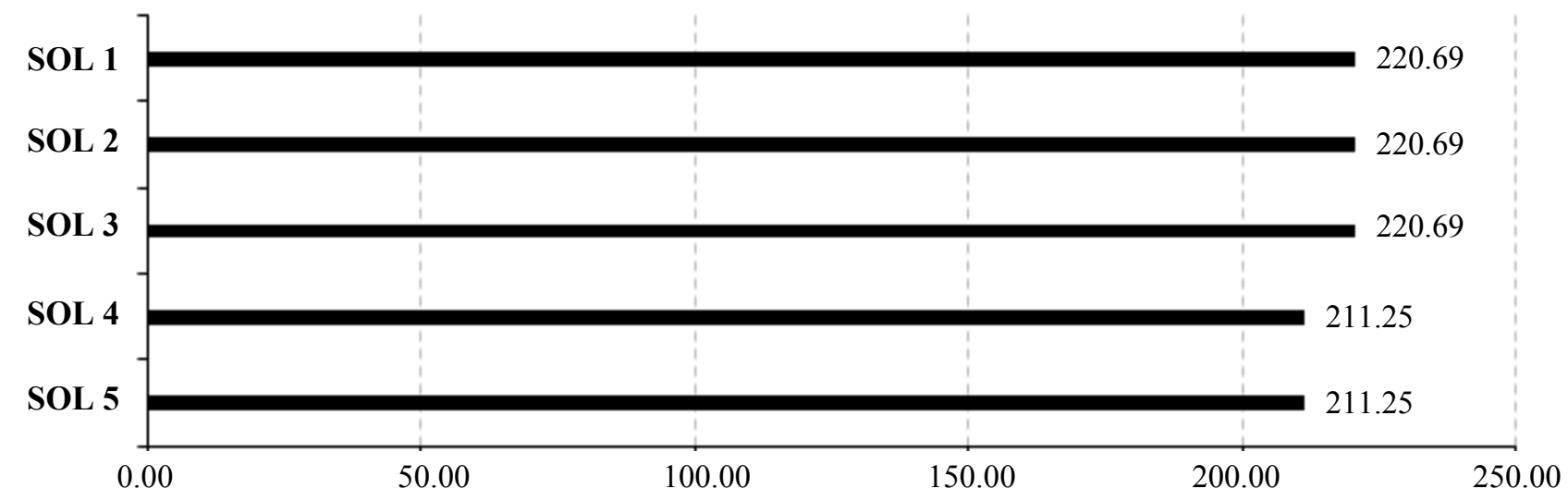

Fig. 3 GWP ( $\mathrm{kg} \mathrm{CO}_{2}$ equivalent).

Global warming potential ( $\mathrm{kg} \mathrm{CO}$ equivalent)

Reference is made to the configuration of the case study (Fig. 1). 


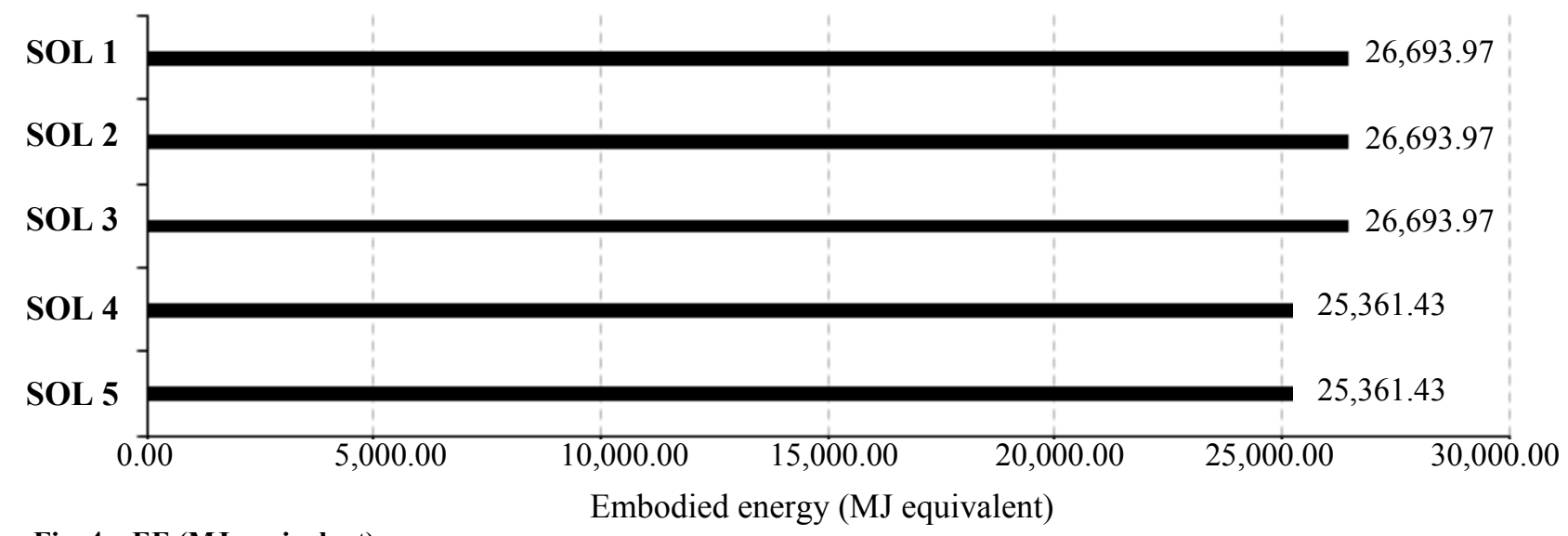

Fig. 4 EE (MJ equivalent).

Reference is made to the configuration of the case study (Fig. 1).

As of the year 2013, external cladding system, in addition to the residual value of the insulating products, includes aluminium profiles whose residual value is upon approximate $0.90 \mathrm{EUR} / \mathrm{t}$ (2012-2013). Residual value changes according to the implemented insulating product. In economic terms, residual value of SEP is much higher (20-100 EUR/t, 100\% recyclable (2012-2013)) compared to that of mineral wool (8\% approx. out of initial investment value, recyclable up to $50 \%$ (2012-2013)).

The state of the art shows that residual value of solutions depending on product recyclability and reusability varies according to the disposal methods implemented in different countries.

Please note that if, for instance, separation of plaster and insulating products upon disposal is carried out as an example in Germany, such practice rarely takes place in Italy. Therefore, the economic variable is of course influenced by market trends, and it is a sensitive system variable to be kept into account upon statistic error correction.

\section{About the Seventh Requirement: Possible Contradictions}

Environmental protection provides specific requirements that are not only technological-related but environmental-related and energy-related.

Such requirements refer to tangible impacts that technological solutions would alternatively have on the community, if selected.

In case of building recovery, we should pay attention to the fact that indicators for the seventh requirement of insulating products might be contrasting.

For instance, pay attention to SEP: SEP in panels is less sustainable than RW (rock wool) and GW (glass wool) but it has more recoverable and more residual value upon dismantling.

Moreover, with reference to initial investments, we consider that it is appealing because it has the lowest cost in terms of initial investments (in particular, as regards supply costs-glass-wool panels are more expensive in terms of supply costs with free delivery to the construction site, followed by rock-wool panels, and, finally, sintered-expanded-polystyrene panels are much cheaper and cost approximately a half compared with the previous ones). A future publication will investigate in-depth such monetary aspects with numerical examples.

In view of environmental sustainability of products, what would happen if we decide to extend from cradle to grave? In monetary terms (economic profits), considering different life stages and the interests of different involved parties, are we always working to guarantee convenience in funding, quality and energy-environmental sustainability?

\section{Conclusions}

The present work has investigated a set of 
technological packages referred to two alternative facade systems - the ETICS and the external cladding system - complying with one thermal-insulation strategy but different in terms of duration of service life.

Reference has been made to maintenance phase and residual value upon dismantling.

Accurate economic assessment requires the entity to be related to periodical checks on performance quality in the building system and in its parts. Therefore, detailed scheduled maintenance activities have been defined.

Based on the research carried out by the author on the Italian market, accurate technical data to draw up future cost estimates and effective appraisals in terms of quality and environmental sustainability have been introduced to the reader.

With reference to maintenance phase, alternative solutions have also been compared by means of environmental indicators.

With reference to environmental sustainability, we consider that the current Italian $^{4}$ and international databank would require further comprehensive implementations, also in view of data compatibility/interoperability.

According to the research, with reference to the seventh requirement of building products, indicators for the seventh requirement might be contrasting, as exemplified in Section 5.

Are we really working to guarantee both quality and environmental sustainability?

In this view, energy quality is not the only thing that counts in a building. Convenience in funding [20] a reference upgrading solution should be assessed based on technological/technical utility [21], cash-flow scenarios and energy-environmental

\footnotetext{
${ }^{4}$ For further information, please refer to the plan agreement between the Italian region of Marche and Itaca, coordinated by ITC-CNR (Construction Technologies Institute-Italian National Research Council) in collaboration with Università Politecnica delle Marche, iiSBE (International Initiative for a Sustainable Built Environment) Italia, Département Energétique et Procédées-DEP (Department Energy and Processes) MINES ParisTech 2B - environmental consulting society, environment park.
}

impacts on the community. Visual comparison of the mentioned aspects may provide for a simultaneous view of a dimensionalised results for specific problems related to different life stages and to different involved stakeholders.

In this view, more burdensome research is needed.

\section{Acknowledgments}

Technical data here presented have been collected/elaborated within a non-market, non-party research carried out by the author autonomously on the Italian market from 2009 to 2013.

Technical data here presented are partially drawn, then at a later stage elaborated, from the already defended Ph.D. thesis [22], where all sources and information used or quoted have been indicated and acknowledged by means of a dedicated reference list.

Special thanks to all professionals and academics who shared experiences with the author within the Ph.D. training period.

\section{References}

[1] H.A. Boussabaine, R.J. Kirkham, Whole Life-Cycle Costing, Risk and Risk Responses, 1st ed., Blackwell Publishing Ltd, Oxford, 2004.

[2] J.W. Bull (Ed.), Life Cycle Costing for Construction, 1st ed., Blackie Academic and Professional, London, 1993.

[3] EMG (Environment Management Group), Report of the Annual Meeting of the Environment Management Group, EMG, Geneva [Online], http://www.unemg.org/Link Click.aspx? fileticket $=$ OgWlwljAEwQ\%3D\&tabid=4014\&1 anguage $=$ en-US (accessed Mar. 23, 2010).

[4] M.E. Burt, A Survey of Quality and Value in Building, Building Research Establishment, Garston, 1978.

[5] B. Atkin, R. Flanagan, L. Marsh, A. Agapiou, Improving Value for Money in Construction: Guidance for Chartered Surveyors and Their Clients, The Royal Institution of Chartered Surveyors, London, 1995.

[6] ASHRAE (The American Society of Heating, Refrigerating and Air Conditioning Engineers) Green Guide, The Design, Construction, and Operation of Sustainable Buildings, 2nd ed., American Society of Heating, Refrigerating and Air-Conditioning Engineers, Inc., Atlanta, 2006.

[7] J.A. Todd, K.M. Fowler, Measuring Performance of Sustainable Buildings, WBDG (Whole Building Design 
Guide), Pacific Northwest National Laboratory [Online] http://www.wbdg.org/resources/measperfsustbldgs.php?r =sustainable (accessed June 28, 2011).

[8] M.T. Andeweg, S. Brunoro, L.G.W. Verhoef (Eds.), Improving the Quality of Existing Urban Building Envelopes-State of the Art, IOS Press, Amsterdam, 2007.

[9] European Organisation for Technical Approval, Guideline for European Technical Approval of External Thermal Insulation Composite System with Rendering, ETAG (European Organisation for Technical Approval), Brussels, 2000.

[10] CORTEXA Handbook, Consorzio Cortexa (Cortexa Consortium), DNArt Studio, Florence, 2009. (in Italian)

[11] Italian Standard Institute, Cladding and Anchoring Systems for Back Ventilated External Enclosures of Buildings, Instructions for the Design, Installation and Maintenance, Ceramic and Stone Cladding, UNI 11018:2003, IHS Standards Expert, 2003.

[12] CPR - Construction Products Regulation No.305/2011, EU Regulations, EU, 2011.

[13] Italian Standard Institute and European Committee for Standardization, Fibre Cement Flat Sheets-Product Specification and Test Methods, UNI EN 12467:2007, HIS (Information Handling Services) Standards Expert, 2007.

[14] R. di Giulio, Manuale di Manutenzione Edilizia. Valutazione del Degrado e Programmazione Della Manutenzione, 3rd ed., Maggioli Editore, Rimini, 2007, pp. 138-142, 223-231. (in Italian)

[15] Italian Standard Institute and European Committee for
Standardization, Maintenance, Maintenance Terminology, UNI EN 13306:2010, IHS Standards Expert, 2010.

[16] Conseil International du Bâtiment (International Council for Building) W86, Building Pathology, A State-Of-The-Art Report, CIB, Delft, the Netherlands, 1993. (in English)

[17] M. Ciampoli, Criteri di Programmazione delle Ispezioni sulle Costruzioni Esistenti e di Interpretazione dei Risultati Sperimentali [Online], http://www.apav.it/sito ratio/file_pdf/ratio_3/capitolo_2.pdf (accessed Aug. 20, 2011). (in Italian)

[18] S.P. Cheong, C.J. Anumba, R. Hill, D. Bouchlaghem, Improving construction client satisfaction through functional briefing, in: Proceedings of Construction Research Congress, Winds of Change: Integration and Innovation in Construction, American Society of Civil Engineers, Reston, 2003, pp. 647-656.

[19] C. Boyle, G.T.K. Coates, Sustainability principles and practice for engineers, IEEE Technology and Society Magazine 24 (3) (2005) 32-39.

[20] D.M. Amidon, Innovation Strategy for the Knowledge Economy: The Ken Awakening, Butterworth-Heinemann, Newton, MA, 1997.

[21] P.N. Maggi, Il processo edilizio, Vol.1—Metodi e Strumenti di Progettazione Edilizia, Polipress, Milan, 1994. (in Italian)

[22] R. Perego, Convenience-assessment path in technological and energy-efficiency upgrading on existing blocks of flats, Ph.D. Thesis, Politecnico di Milano, Department of Building Environment Science and Technology, Milan, 2012. 